\title{
A speculative outlook on embryonic aneuploidy: Can molecular pathways be involved?
}

\author{
Olga Tšuiko $^{\mathrm{a}, \mathrm{b}}$, Tatjana Jatsenko ${ }^{\mathrm{b}}$, Lalit Kumar Parameswaran Grace ${ }^{\mathrm{c}}$, Ants Kurg ${ }^{\mathrm{d}}$, \\ Joris Robert Vermeesch ${ }^{\mathrm{e}}$, Fredrik Lanner ${ }^{\mathrm{f}}$, Signe Altmäe ${ }^{\mathrm{b}, \mathrm{g}, *}$, Andres Salumets $^{\mathrm{a}, \mathrm{b}, \mathrm{h}, \mathrm{i}}$ \\ a Department of Biomedicine, Institute of Bio- and Translational Medicine, University of Tartu, Tartu 50411, Estonia \\ b Competence Centre on Health Technologies, Tartu 50410, Estonia \\ c Department of Women's and Children's Health, Division of Obstetrics and Gynecology, Karolinska Institutet, Karolinska University Hospital, Stockholm \\ 17176, Sweden \\ d Department of Biotechnology, Institute of Molecular and Cell Biology, University of Tartu, Tartu 51010, Estonia \\ e Laboratory of Cytogenetics and Genome Research, Center of Human Genetics, KU Leuven, Leuven 3000, Belgium \\ ${ }^{\mathrm{f}}$ Department of Clinical Science, Intervention, and Technology, Karolinska Institutet, Stockholm 14186, Sweden \\ g Department of Biochemistry and Molecular Biology, Faculty of Sciences, University of Granada, Granada 18071, Spain \\ ${ }^{\text {h }}$ Department of Obstetrics and Gynecology, Institute of Clinical Medicine, University of Tartu, Tartu 51014, Estonia \\ ${ }^{i}$ Department of Obstetrics and Gynecology, University of Helsinki and Helsinki University Hospital, Helsinki 00029, Finland
}

\section{A R T I C L E I N F O}

\section{Keywords:}

Aneuploidy

Chromosomal instability

Molecular signaling

Preimplantation embryo

\begin{abstract}
A B S T R A C T
The journey of embryonic development starts at oocyte fertilization, which triggers a complex cascade of events and cellular pathways that guide early embryogenesis. Recent technological advances have greatly expanded our knowledge of cleavage-stage embryo development, which is characterized by an increased rate of wholechromosome losses and gains, mixoploidy, and atypical cleavage morphokinetics. Embryonic aneuploidy significantly contributes to implantation failure, spontaneous miscarriage, stillbirth or congenital birth defects in both natural and assisted human reproduction. Essentially, early embryo development is strongly determined by maternal factors. Owing to considerable limitations associated with human oocyte and embryo research, the use of animal models is inevitable. However, cellular and molecular mechanisms driving the error-prone early stages of development are still poorly described. In this review, we describe known events that lead to aneuploidy in mammalian oocytes and preimplantation embryos. As the processes of oocyte and embryo development are rigorously regulated by multiple signal-transduction pathways, we explore the putative role of signaling pathways in genomic integrity maintenance. Based on the existing evidence from human and animal data, we investigate whether critical early developmental pathways, like Wnt, Hippo and MAPK, together with distinct DNA damage response and DNA repair pathways can be associated with embryo genomic instability, a question that has, so far, remained largely unexplored.
\end{abstract}

\section{Introduction}

Embryonic aneuploidy is a major factor contributing to implantation failure, spontaneous miscarriage and low in vitro fertilization (IVF) success rate. Aneuploidy burden at early cleavage-stages in human is also associated with developmental arrest at the time of embryonic genome activation (EGA) (Maurer et al., 2015). The advent of powerful single-cell technologies and time-lapse imaging has provided novel insights into mechanistic origins of chromosome imbalances in human preimplantation development (Fig. 1). Sophisticated single-cell assays uncovered that $70-80 \%$ of human IVF cleavage-stage embryos is made up of cells bearing signatures of chromosomal instability (CIN), which is a hallmark of tumorigenesis, characterized by an increased rate of whole or segmental chromosome aberrations (Chavez et al., 2012; Chow et al., 2014; Mertzanidou et al., 2012; Vanneste et al., 2009; Voet et al., 2011a; Zamani Esteki et al., 2015). Time-lapse imaging has revealed that chromosome imbalances in human IVF embryos are not always underlined only by mitotic errors, such as anaphase lagging and chromosome nondisjunction, but are often accompanied by aberrant cleavage patterns with or without cellular fragmentation. Atypical cell morphokinetics, such as entire blastomere fusion or failure of cytokinesis, significantly contribute to genomic instability and can be observed in $>50 \%$ of aneuploid embryos (Chavez et al., 2012; Hardy et al., 1993). Recently, fusion of a second

\footnotetext{
* Corresponding author at: Department of Biochemistry and Molecular Biology, Faculty of Sciences, University of Granada, Granada 18071, Spain.

E-mail address: signealtmae@ugr.es (S. Altmäe).
} 


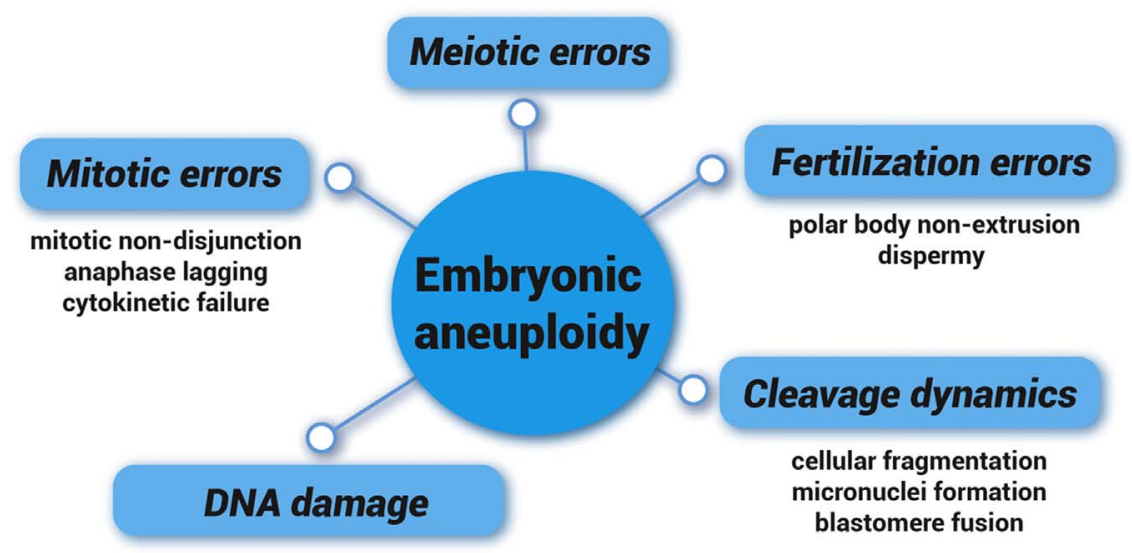

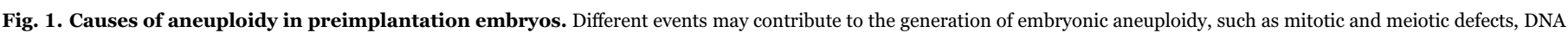

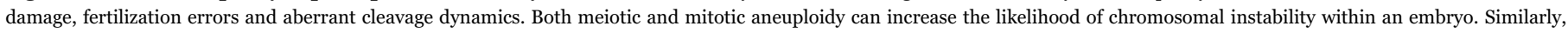
aberrant fertilization and impaired cleavage morphokinetics can lead to blastomeres with unstable genomes or chaotic chromosome patterns.

polar body was detected by whole-genome haplotyping of trophectoderm (TE) biopsy derived from human IVF blastocyst (Ottolini et al., 2015). The persistence of polar body DNA in the embryo until the blastocyst stage was rather unexpected, giving the fact that polar bodies undergo fragmentation after extrusion from the oocyte upon fertilization. Also, a novel phenomenon of spontaneous parental genome segregation in the zygote was discovered in bovine in vitro embryos, demonstrating that paternal and maternal genomes can be separated into distinct androgenetic (carrying only paternal genome) and gynogenetic (carrying only maternal genome) cell lineages (Destouni et al., 2016), likely giving rise to chimaerism and mixoploidy, observed in humans (Edwards et al., 1994; Jarvela et al., 1993; Yamazawa et al., 2010). Taken together, all these data reveal the remarkable genome plasticity of preimplantation development.

Different studies on animal models have demonstrated that aneuploidy and mosaicism in early embryos are not restricted only to humans, but have also been observed in farm animals (Destouni et al., 2016; Rambags et al., 2005; Viuff et al., 2000; Zudova et al., 2003) and non-human primates (Dupont et al., 2009, 2010), indicating that they may serve as appropriate animal models for studying genomic instability in embryos. For instance, by using bovine as a model, we and Viuff et al. demonstrated that in vitro environment can affect the frequency and nature of chromosome anomalies in cleavage-stage embryos (Tsuiko et al., 2017; Viuff et al., 2001). In contrast, spontaneous aneuploidy rate in mouse embryos is very low, thus chromosome segregation errors in mice are usually induced by using different inhibitors (Bolton et al., 2016; Lightfoot et al., 2006). Using this approach, a recent study in mice investigated the developmental fate of induced mosaic aneuploidies and demonstrated that aneuploid cells become progressively depleted from the embryo at later stages of development (Bolton et al., 2016). This finding together with the fact that mosaic embryos can successfully result in live birth of healthy babies in human (Greco et al., 2015) challenges the hypothesis that aneuploid embryos are not as developmentally competent as their euploid counterparts. At the same time, these observations highlight our limited knowledge on the molecular causes and the developmental fate of human embryonic CIN and raise important questions regarding both the burden and nature of aneuploidy that can be tolerated throughout development.

Essentially, preimplantation development processes are rigorously regulated by multiple signal-transduction pathways that drive cell division and cellular growth, differentiation, migration and apoptosis (Zhang et al., 2007). Interestingly, profound research in the field of developmental biology and cancer revealed that early embryo development shares many similarities with tumorigenesis in terms of cell proliferation and differentiation mechanisms, as well as similarities in signal transduction pathways (Ma et al., 2010). In addition, molecular pathways crucial for oogenesis and embryo development, such as DNA damage response (DDR), Wnt, Hippo and MAPK are also driving CIN in cancers, indicating their role in cell cycle progression (Orr and Compton, 2013). Moreover, recent studies on mouse embryonic stem cells (ESCs) also demonstrated that altered Wnt or MAPK signaling leads to impaired cellular differentiation, genomic instability and reduced survival of ESCs (Augustin et al., 2017; Chen et al., 2015). However, the role of signaling pathways in maintaining genomic stability in oogenesis and early embryo development remains elusive. Therefore, investigating the potential link between signaling pathways and embryonic aneuploidy may uncover critical aspects of fundamental developmental processes.

Here, we summarize the origins of aneuploidy in early embryos and explore how embryonic DNA damage response pathway operates to ensure embryo survival through preimplantation period. We also focus on conserved signaling pathways crucial for oocyte and embryo development, such as Wnt, MAPK, and Hippo, which have all been implicated in mitotic progression and/or reduced developmental potential of ESCs. By reviewing the data from human and animal studies, we speculate whether these pathways can be implicated in preimplantation genome stability maintenance and if they can be attributed to embryonic aneuploidy.

\section{Origins of aneuploidy in preimplantation embryos}

\subsection{Meiotic origin of embryonic aneuploidy}

Meiosis is one of the defining milestones of human gametogenesis, which involves two sequential cell divisions, meiosis I (MI) and meiosis II (MII), that result in the formation of haploid gametes. Genome integrity of germ cells strongly relies on intact cellular and molecular mechanisms, involved in accurate chromosome segregation during meiotic progression. Key meiotic events guiding this specialized process have been recently described elsewhere (Ohkura, 2015). It is worth noting that a number of sex-specific differences make female meiosis more complex. The most obvious difference occurs already at the late MI prophase: male gametes proceed quickly through the first meiotic division, while oocytes encounter meiotic arrest that can last for decades. Second, unlike developing spermatocytes, oocytes lose their centrioles during meiotic prophase and subsequently segregate their chromosomes on an acentriolar spindle (Schuh and Ellenberg, 2007). Finally, male germ divisions are symmetric and produce four equally sized gametes, while eccentric positioning of oocyte meiotic spindle results in an asymmetric cell division that yields a large fertilizable egg and three or four small non-functional polar bodies 
(Brunet and Verlhac, 2011). Such asymmetric division also allows the oocyte to retain maximum of it cellular cytoplasm that contains mRNA, proteins and nutrients necessary for achieving oocyte and embryonic developmental competence.

Errors in either first or second meiotic division lead to zygotic inheritance of meiotic-based aneuploidies that can consequently cause spontaneous miscarriages, stillbirth or congenital birth defects (Hassold and Hunt, 2001). For example, defects in crossover sites, or chiasmata, assembly, maintenance and function in both male and female gametes can lead to failure of proper homolog disjunction in meiosis I (Fledel-Alon et al., 2009; Hirose et al., 2011). Notably, meiotic errors in humans are mostly maternal in origin and arise during the complex stages of oogenesis (reviewed in Webster and Schuh, 2016). In addition, male cell cycle checkpoint mechanisms seem to be more robust and can successfully eliminate cells with meiotic errors regardless of paternal age (Vrooman et al., 2014). In contrast, female spindle assembly checkpoint (SAC) mechanisms appear to be weaker and are activated only in the presence of several misaligned chromosomes, but they are not able to block meiotic progression, when only a single chromosome fails to align properly (Nagaoka et al., 2011). Such oocyte-specific SAC-mediated control of meiosis can partially explain, why oocytes are more prone to aneuploidy than sperm (Sun and Kim, 2012). Moreover, the incidence of maternal aneuploidy drastically increases in women along with age, with lowest aneuploidy rate observed in women between the ages of 26 and 30 (20-27\%), while aneuploidy rate reaches up to $80 \%$ by the age 45 (Franasiak et al., 2014; Munne et al., 1995). One of the reasons for this can be the agerelated decrease in the ability to maintain proper spindle checkpoint signaling and compromised cohesion between sister chromatids (Yun et al., 2014). The level of cohesin is significantly reduced in both human and mouse aged oocytes and it is not capable anymore to stabilize chiasmata in oocytes or hold sister chromatids together (Chiang et al., 2011; Tsutsumi et al., 2014). It was shown in mice that the age-related loss of cohesin may be associated with the depletion of Shugoshin proteins that protect centromeric cohesin from cleavage and premature segregation of sister chromatids (Lister et al., 2010). In addition, age-related defective spindle assembly can be attributed to the chaotic microtubule dynamics, resulting in multipolar spindle formation that predisposes the oocyte to chromosome segregation errors (Nakagawa and FitzHarris, 2017). Importantly, when fertilized, aneuploid eggs will unavoidably give rise to aneuploid embryos that will most likely fail to survive.

\subsection{Mitotic origin of embryonic aneuploidy}

Up to $80 \%$ of chromosome imbalances in human IVF embryos are mitotic in origin and lead to chromosomal mosaicism (the presence of cells with different genomic content in one organism). In contrast to meiotic errors, mitotic aberrations are age-independent and can arise also in embryos from young fertile couples (Vanneste et al., 2009). Accurate post-zygotic mitotic division also relies on the intact assembly of mitotic spindle apparatus and the integrity of SAC to ensure proper separation and segregation of chromosomes to daughter cells. Prior to the formation of the first mitotic spindle in zygotes, microtubuledependent movement of maternal and paternal pronuclei towards each other takes place. In cattle and humans, the paternally inherited centrosome nucleates microtubules to form the sperm aster, which, upon expansion, allows the female pronucleus to associate with microtubules and move towards the male pronucleus in a dyneindependent manner (Clift and Schuh, 2013). Subsequently, the sperm centrosome generates the mitotic spindle that segregates chromosomes to two sister blastomeres during the first post-zygotic division. However, in some fertilized oocytes the first post-zygotic cleavage does not occur as expected. Under in vitro conditions almost $12 \%$ of human zygotes divide into three cells (Chamayou et al., 2013). Time-lapse analysis of human IVF embryos has revealed that tripolar division is frequent in diandric or digynic zygotes with three pronuclei (3PN), carrying either an extra set of haploid paternal or maternal chromosomes, respectively (Staessen and Van Steirteghem, 1997), but bipronuclear (2PN) embryos also exhibit abnormal first post-zygotic cleavage (Hlinka et al., 2012). In addition, it has been hypothesized that gonomeric spindle formation and/or asynchronous parental cell cycles within the zygote can lead to the generation of separate gynogenetic and androgenetic cells upon first post-zygotic cleavage (Destouni et al., 2016; Destouni and Vermeesch, 2017).

As embryonic cleavage proceeds, errors in post-zygotic division, such as centrosome overduplication, impaired spindle assembly, chromosome cohesion/segregation dynamics and cytokinesis, can contribute to chromosome mis-segregation and aneuploidy (Silkworth and Cimini, 2012). Indeed, knockout studies in mouse pre- and peri-implantation embryos have revealed that disruptions in key genes involved in the centrosome/kinetochore structure or cellcycle checkpoints lead to aneuploidy, mitotic arrest, abnormal mitotic division and early embryonic lethality (Artus et al., 2006). Although there are numerous possible mechanisms leading to mitotic errors in cleavage embryos (reviewed in (Mantikou et al., 2012)), anaphase lagging and mitotic non-disjunction are the most frequent causes of embryonic aneuploidy and mosaicism (Fig. 2A). During mitotic nondisjunction, chromatids fail to separate at the centromere during cell division, resulting in loss of a chromosome in one daughter cell and a reciprocal gain in the other. In contrast to chromosome non-disjunction, anaphase lagging occurs when one of the sister chromatids fails to connect to the spindle apparatus or is prematurely dissociated from it and is lost upon cytokinesis, resulting in one diploid daughter cell and one cell with a monosomy. In addition, erroneous merotelic kinetochore-spindle microtubule (k-MT) attachments can also contribute to the onset of aneuploidy and CIN in early embryos. Merotelic k-MT attachment occurs when single kinetochore simultaneously attaches microtubules emerging from opposite spindle poles (Cimini et al., 2001) (Fig. 2B). It has been proposed that merotely can also induce centromere-localized DNA breaks leading to sub-chromosomal imbalances that can be accompanied by breakage-fusion-bridge cycle and/or result in complex genomic rearrangements observed in human IVF embryos (Vanneste et al., 2009; Voet et al., 2011a, 2011b). Because kinetochores are attached by MTs from both sides and support chromosome alignment on a metaphase plate, merotely avoids detection by SAC, which leads to failure of chromatid segregation and lag of one chromosome near the central spindle, while the other chromosome moves towards the spindle pole (Thompson and Compton, 2011). Consequently, the lagging chromosomes can form micronuclei in the cytoplasm, providing the basis for cellular fragmentation in human embryos (Chavez et al., 2012). Once trapped within micronuclei, chromosomes can undergo defective DNA replication, resulting in DNA damage and frequent double-strand breaks (DSB) (Crasta et al., 2012). In turn, DSB can trigger an error-prone non-homologous endjoining (NHEJ) repair mechanism in micronuclei (Ly et al., 2017) that can result in chromosomal aberrations. These events may also lead to chromosome pulverization within the micronuclei. Moreover, chromosomal fragments can be incorporated back into the genome of a developing embryo upon fusion of the micronuclei with blastomeres, resulting in chaotic chromosome aberration patterns (Chavez et al., 2012).

\section{Can early developmental signaling be linked to embryonic aneuploidy?}

The underlying molecular causes responsible for error-prone embryo cleavage are largely unknown, as the mechanisms of chromosome segregation in preimplantation embryos are still poorly described. Extensive research in the cancer field shed some light into the key signaling pathways that are dysregulated during tumorigenesis, indicating their potential involvement in maintaining genomic stability 


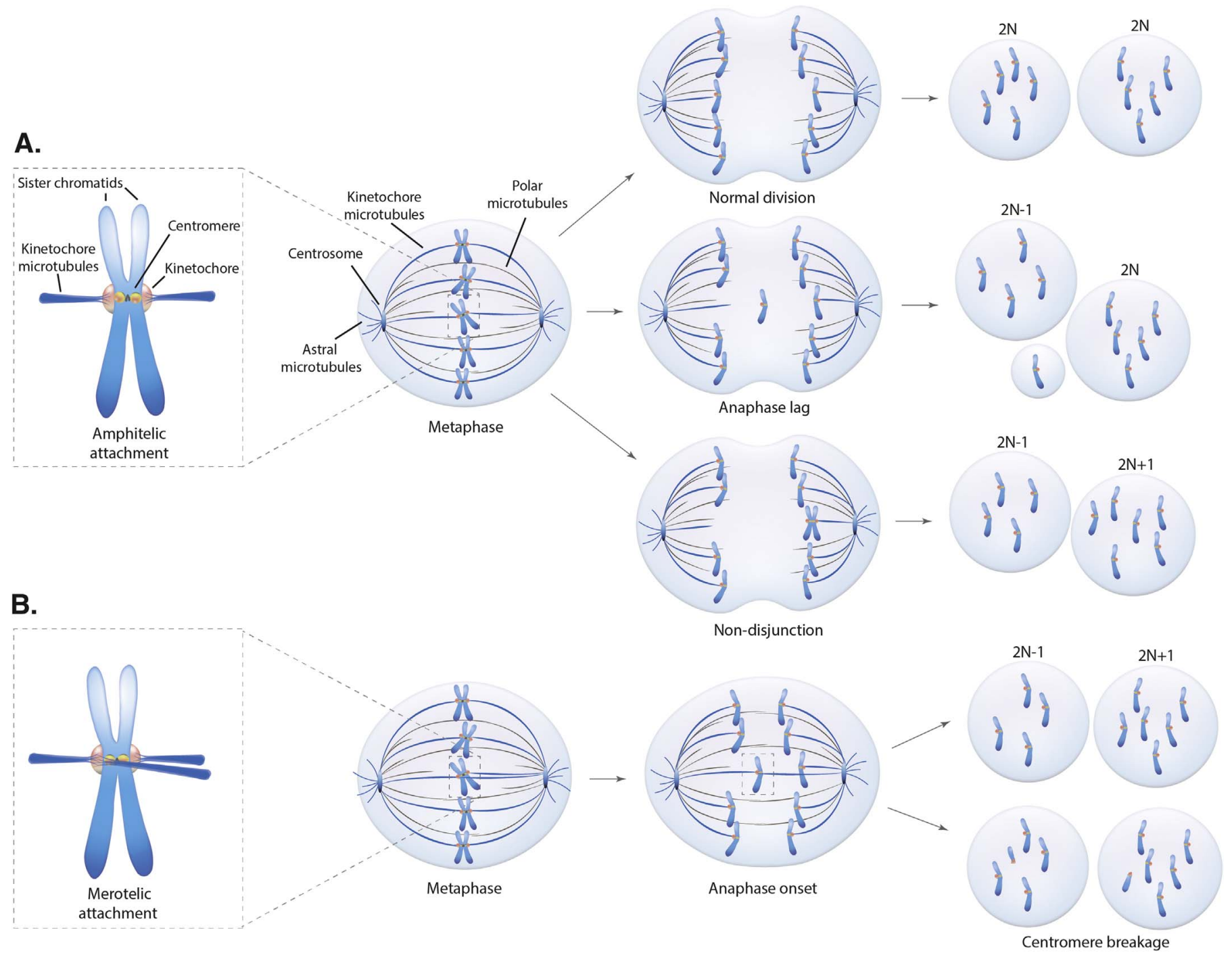

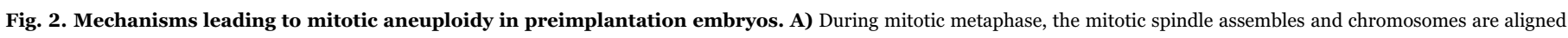

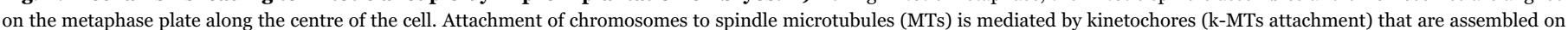

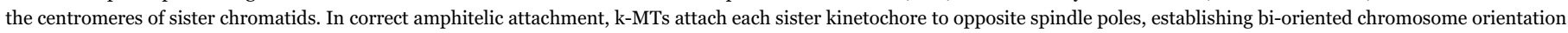

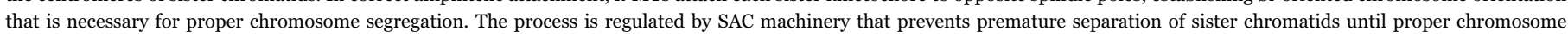

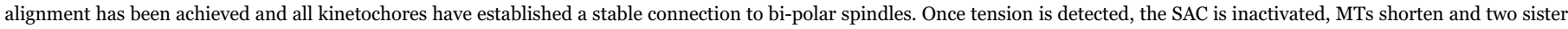

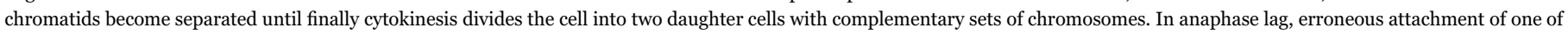

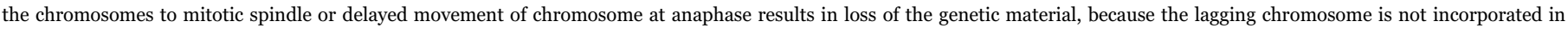

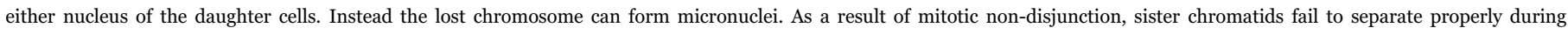

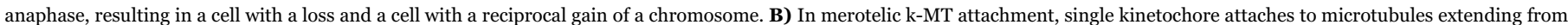

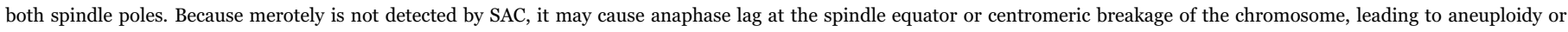
complex sub-chromosomal rearrangements, respectively.

(Orr and Compton, 2013). Therefore, it is tempting to speculate whether aberrant molecular signaling may play a role in embryonic aneuploidy formation and whether findings from cancer research can be translated to early embryogenesis.

\subsection{The DNA damage response and repair pathway}

The mechanisms of DDR and DNA repair have essential roles in maintaining genome stability and its function. In somatic cells, the DDR pathway is a tightly coordinated signal transduction network, involving cell-cycle checkpoint activation and DNA damage repair and tolerance mechanisms via ATM- and ATR-mediated pathways (Smith et al., 2010). ATM and ATR are the most upstream kinases of DDR signaling that activate cell-cycle checkpoint proteins CHK1/CHK2 and DNA repair or apoptotic mechanisms in response to DNA damage (Marechal and Zou, 2013) (Fig. 3). CHK1 has also been implicated in mitotic regulations in several ways: (i) it is necessary for centrosome amplification upon DNA damage, (ii) it regulates spindle function by participating in establishment of correct k-MT attachment and (iii) it phosphorylates SAC components and ensures proper chromosome segregation and cytokinesis (Bourke et al., 2007; Chila et al., 2013; Peddibhotla et al., 2009; Zachos et al., 2007).

DDR pathway represents one of the most intriguing aspects of preimplantation development. In contrast to somatic cells, the regulation of DDR pathways and downstream action in preimplantation embryos seem to vary at different stages of development, potentially making them more vulnerable to chromosome segregation errors (Jaroudi and SenGupta, 2007). First, some studies on mouse and porcine embryos suggest that DNA damage checkpoint and repair may be insufficient in embryos prior to EGA possibly due to limited ATM kinase activity (Adiga et al., 2007; Wang et al., 2015). In addition to cell-cycle checkpoint activation, ATM phosphorylates histone H2AX 


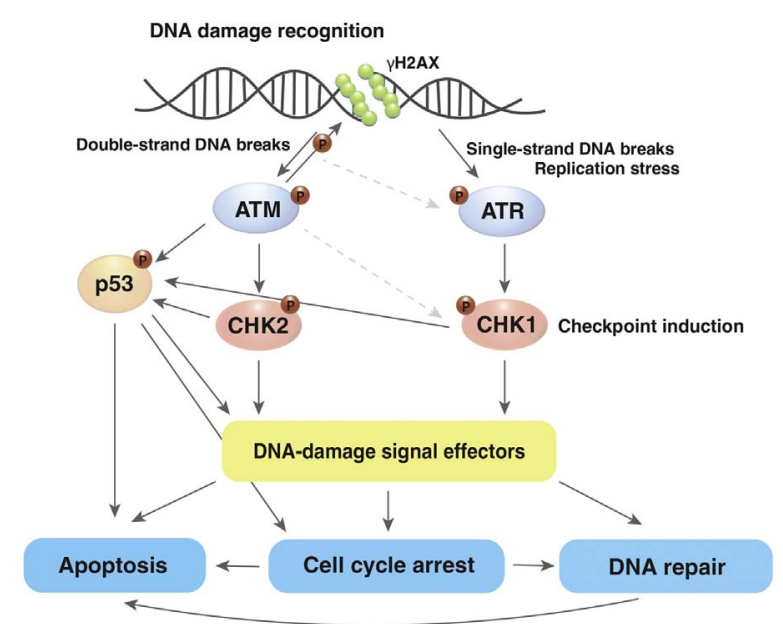

Fig. 3. Simplified representation of the DNA damage response pathway. DNA damage- and replication stress-induced double-strand and single-strand DNA breaks activate ATM and ATR kinases, respectively. ATM and ATR are key signal transducers of downstream DDR pathways. Once phosphorylated, they trigger the activation of downstream cell-cycle regulators CHK1 and CHK2, which in turn signal downstream checkpoints that stop cell-cycle progression and activate DNA damage repair and tolerance mechanisms. ATM also phosphorylates H2AX and amplifies DNA damage signal. In addition, DDR induces the activation of tumor suppressor p53, a critical sensor of DNA damage, which determines cell fate depending on the levels of DNA damage or DNA-repair efficiency.

$(\gamma \mathrm{H} 2 \mathrm{AX})$ at the site of DSB that recruit necessary DNA repair factors (Celeste et al., 2002; Paull et al., 2000). However, it was demonstrated that loss of ATM activity in early mouse embryos and embryonic stem cells compromised phosphorylation of downstream targets necessary for DNA DSB repair, consequently leading to proliferation defects and genomic instability due to chromatid breaks (Yamamoto et al., 2012). Therefore, if embryos initially lack proper ATM activity, it may partially explain, why first post-zygotic cell divisions are error-prone: in the checkpoint-compromised background, DNA and chromosome damage that results in mitotic failure may be propagated to the next cell cycle, exacerbating chromosome aberrations within the genome (Hayashi and Karlseder, 2013).

Second, upon massive DNA damage DDR in somatic cells triggers apoptosis to eliminate damaged cells, however functional apoptotic pathways seem to be suppressed in early human and bovine cleavagestage embryos (Bazrgar et al., 2014; Fear and Hansen, 2011). In murine zygotes maternal and paternal pronuclei also respond to severe sperm DNA damage through a unique non-apoptotic pathway that delays paternal nuclei replication and provides time to repair DNA damage (Gawecka et al., 2013). In addition, although SAC is active in early embryos, it is not able to drive SAC-induced apoptosis at cleavage-stages of development (Jacobs et al., 2017). The absence of early apoptotic response suggests that blastomeres with damaged DNA or aberrant chromosomes are not eliminated during the first postzygotic divisions, thus facilitating the survival of the embryo until later stages. As such, it was also proposed that embryonic cell cycle checkpoints and regulators may be more permissive, allowing rapid cleavage divisions. For instance, some of the essential G1 and G2 cell checkpoint proteins, like RB and WEE1, are silenced in 8-cell human embryos and are only activated at later stages of development, suggesting that blastocyst have active cell cycle checkpoints that ensure chromosome integrity of the developing embryo (Kiessling, 2010; Kiessling et al., 2009, 2010). Therefore, cells with damaged or incompletely replicated DNA or improper chromosome alignment can still proceed to mitosis, bypass SAC-mediated arrest and continue cell division, leading to increased rates of chromosomal aberrations and aneuploidy during the first cleavages of early embryos. Consistent with the idea of permissive checkpoints in the early embryo, a subset of differentially expressed mitotic genes was identified in human aneu- ploid embryos compared to euploid ones, including SAC components $B U B 1$ and BUB3, as well as PTTG1, which prevents premature sister chromatid separation, and the cell-cycle regulator TP53 (VeraRodriguez et al., 2015). Moreover, many of the differentially expressed genes were maternal in origin, suggesting that an inherited altered transcriptome in human zygotes may potentially lead to altered first cleavage and contribute to propagation of mitotic aneuploidies in early embryos. These results indirectly support the concept that human embryos rely on maternal resources to recognize DNA damage and activate repair mechanisms, ensuring embryo survival during first postzygotic cleavages. However, mammalian oocytes, especially human, can be surprisingly inefficient in responding to DNA damage (Carroll and Marangos, 2013; Wang et al., 2017), which may potentially explain the poor oocyte competence to maintain genomic stability in early embryos prior to EGA. Moreover, DNA repair efficiency significantly drops in aged mammalian oocytes derived from mice, human and non-human primates due to impaired ATM-mediated DDR (Titus et al., 2013; Zhang et al., 2015).

Finally, paternal and maternal genome of mammalian zygotes have a different chromatin configuration (Burton and Torres-Padilla, 2014). Epigenetic asymmetry in the human zygote makes paternal chromosomes more susceptible to kinetochore attachment and mis-segregation errors (van de Werken et al., 2015). Interestingly, a recent study in mice uncovered that DNA damage repair pathways are also actively involved in zygotic reprogramming of the paternal genome (Ladstatter and Tachibana-Konwalski, 2016). The authors discovered a CHK1mediated zygotic checkpoint that monitors demethylation-associated DNA lesions on the paternal genome in zygotes and prevents it from entering into mitosis in the presence of unrepaired DNA damage. This observation enforces previous findings, demonstrating that depletion of CHK1 function in mouse embryos leads to the formation of abnormal nuclei and failure to activate cell cycle arrest before mitosis upon DNA damage and replication block (Liu et al., 2000; Takai et al., 2000). Consequently, unresolved DNA damage may impair centrosome integrity, resulting in multipolarity and chromosome missegregation during mitotic divisions (Hut et al., 2003).

Despite the safeguarding role of the DDR, its activation during mitosis was unexpectedly found to induce errors in chromosome segregation in somatic cancer cells by hyperstabilizing k-MT attachments (Bakhoum et al., 2014), making the role of DDR in mitotic processes a topic of intense investigation (Hayashi and Karlseder, 2013). However, because embryos have a distinct DNA damage coping mechanism compared to somatic cells, further research is warranted to reveal, whether DDR activation can interfere with mitotic fidelity in early embryogenesis.

\subsection{The Wnt signaling pathway}

The Wnt signaling pathway plays a critical role in embryo axis formation, cell migration and differentiation, maintenance of cell pluripotency and subsequent embryonic and placental development (Altmäe et al., 2010; Horcajadas et al., 2007; Knofler and Pollheimer, 2013; Tepekoy et al., 2015). The Wnt signaling includes Wnt ligands, G-protein-coupled Frizzled (FZD) family receptors and lipoprotein receptor-related proteins (LRPs) (Miller, 2002), and operates via three different pathways: the canonical Wnt/ $\beta$-catenin pathway, the noncanonical $\mathrm{Wnt} / \mathrm{Ca}^{+}$pathway and the non-canonical planar cell polarity pathway (Komiya and Habas, 2008). The most studied pathway is the canonical $\beta$-catenin-dependent signaling pathway, which regulates the cytoplasmic accumulation of $\beta$-catenin and its nuclear translocation and activity (Fig. 4) (reviewed in MacDonald et al., 2009).

Many of the Wnt components are expressed in mouse and human oocytes and early embryos (Assou et al., 2010; Harwood et al., 2008; Wang et al., 2004). It has been shown that Wnt-mediated signaling in mice coordinates germ cell entry into meiosis and presumably follicular maturation (Naillat et al., 2010). In addition, a meta-analysis of bovine, 
A.

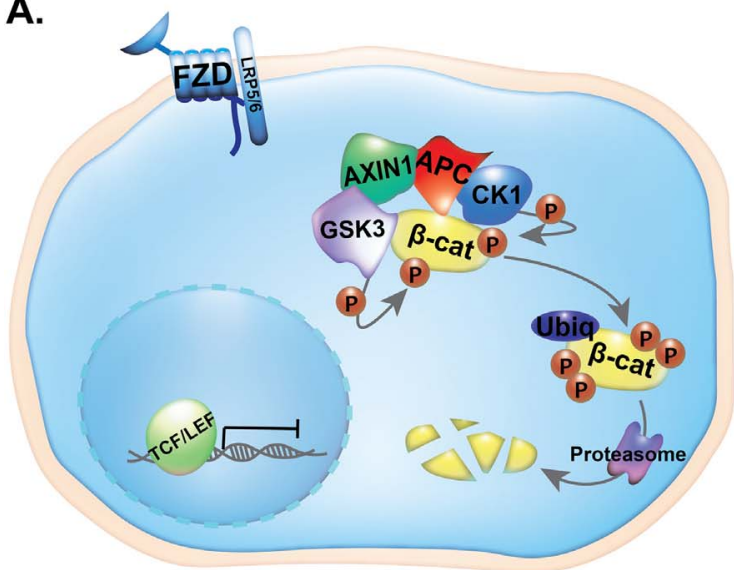

B.

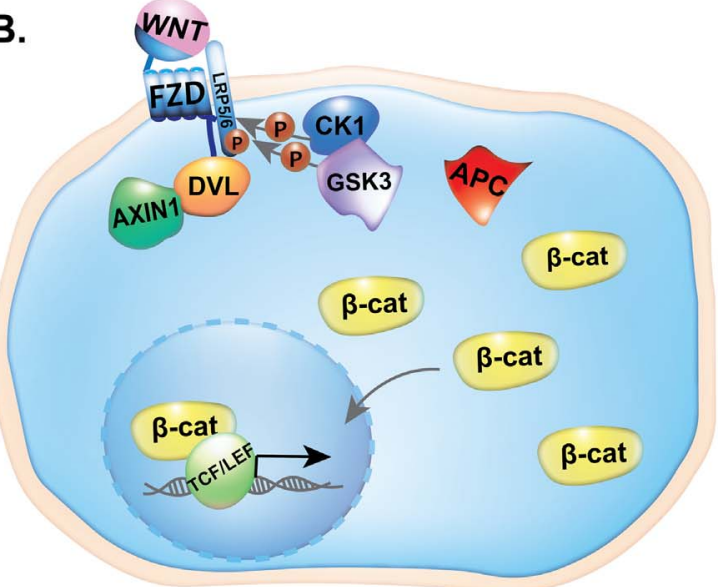

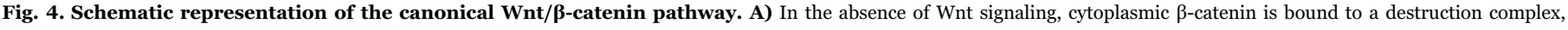

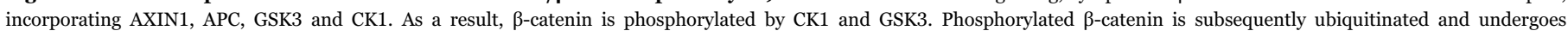

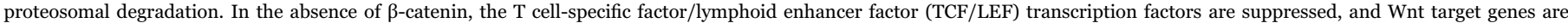

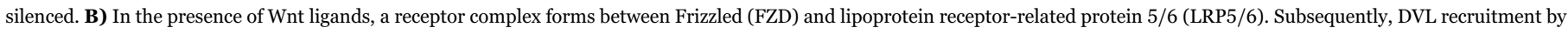

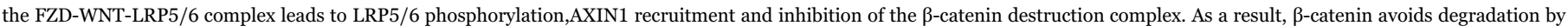

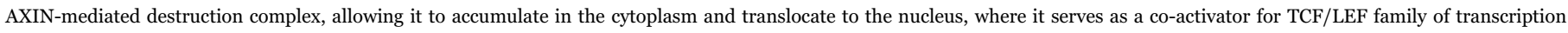
factors to regulate Wnt target genes.

mouse and human oocytes suggested that negative regulation of Wnt decreases oocyte quality, which in turn can adversely affect early embryonic development (O'Shea et al., 2012). At the same time, the Wnt pathway in oocytes seems to be regulated in timely fashion: the canonical Wnt signaling in mouse oocytes was shown to be increasingly activated from the secondary stage of follicular development until ovulation, when Wnt signal becomes depleted (Usongo et al., 2012). In contrast, ovulated oocytes with still active Wnt signal transduction have abnormal morphology and show signs of cytoplasmic fragmentation. Such oocyte cytoplasmic fragmentation can deplete early embryo of key molecules, compromisingits developmental potential. It is worth noting that more than $80 \%$ of human IVF embryos exhibit some degree of fragmentation after first cell divisions, which is mostly of maternal origin (Han et al., 2010; Salumets et al., 2002). Because Wnt components seem to have an important role in ovarian folliculogenesis (Hernandez Gifford, 2015) and its dysregulation was associated with decreased oocyte quality, it might be plausible that proper regulation of Wnt pathway also determines oocyte cytoplasmic maturation, which is necessary to meet the demands of a cleaving embryo until EGA and support its survival until later stages of embryogenesis (Stensen et al., 2014).

In addition to regulating gene transcription, numerous Wnt components have been implicated in mitotic progression in somatic cells by modulating microtubule dynamics, spindle formation and centrosome division. In particular, various key Wnt players, such as the scaffolding protein disheveled (DVL), AXIN1, AXIN2/conductin, GSK3, $\beta$-catenin and APC are all localized on various mitotic structures, where they might have specific functions (Hadjihannas et al., 2006; Niehrs and Acebron, 2012) (Fig. 5A-C). For example, both $\beta$-catenin and GSK3 are implicated in microtubule growth and centrosome separation (Valenta et al., 2012; Wakefield et al., 2003). DVL was shown to localize to centrosomes during mitosis, where it is required for spindle orientation and stable k-MT attachment upon phosphorylation by negative Wnt/ $\beta$-catenin signaling regulator, polo-like kinase 1 (PLK1) (Fig. 5B) (Kikuchi et al., 2010). In addition, DVL can recruit SAC components MPS1, BUB1 and BUBR1 to kinetochores (Fig. 5C) (Kikuchi et al., 2010). AXIN1 and AXIN2 are also localized on the centrosomes, where PLK1 dynamically phosphorylates AXIN1 that might play a role in microtubule stabilization (Ruan et al., 2012), while AXIN2 appears to be involved in regulation of centrosome cohesion (Hadjihannas et al., 2010). Finally, APC is localized to the mitotic spindle and ensures proper chromosome segregation by correcting kMT attachment errors (Fodde et al., 2001; Kaplan et al., 2001).

In parallel to somatic cells, Wnt pathway components might also be involved in regulation of cell cycle progression in oocytes and early embryos. For instance, GSK3 inhibitors induced abnormal spindle formation and chromatin segregation during the first post-zygotic cleavage in mouse zygotes, and such embryos became arrested at the two-cell stage (Acevedo et al., 2007). Downregulation of Axin-1 during meiotic maturation of mouse oocytes leads to defective spindle organization, impaired pronuclear formation and first polar body extrusion (He et al., 2016). Both APC and DVL are also highly expressed in human MII oocytes, where they may potentially fulfill the role of stabilizers of k-MT attachments throughout MII arrest (Wells et al., 2005). Lastly, PLK1 was associated with accurate spindle assembly and chromosome segregation during prometaphase stage of the first post-zygotic divisions in porcine embryos (Zhang et al., 2017).

While it is well established that multiple Wnt pathway components play an important role during mitosis, there is accumulating evidence that they can execute mitotic regulation in a Wnt-independent matter. In addition, how can Wnt signaling cascade function in mitotic spindle and k-MT formation, when the transcription machinery is shut off? A potential answer to that question was provided by an elegant study in HeLa cell lines, demonstrating that Wnt-dependent stabilization of proteins (Wnt/STOP), which peaks at G2/M transition and mitosis, occurs in a $\beta$-catenin-independent manner (Acebron et al., 2014). It was established that Wnt/STOP is required for proper microtubule dynamics within the mitotic spindle and faithful chromosome segregation. Wnt/STOP seems to be regulated by basal Wnt signaling, as repression of $L R P 5 / 6$ or $D V L$ led to erroneous k-MT attachments, resulting in abnormal spindle formation and chromosome lagging (Stolz et al., 2015). Based on these novel findings, it cannot be excluded that Wnt/STOP might also control other regulatory steps during mitotic division, but the clear function of this pathway remains to be elucidated. Intriguingly, Wnt/STOP appears to be active in MII arrested Xenopus oocytes, where it might be involved in stabilization of proteins required for subsequent embryonic division, as inhibition of maternal Wnt/STOP signaling in oocytes led to cleavage arrest after fertilization (Huang et al., 2015). In addition, because mammalian cleavage-stage embryos often exhibit aneuploidy, one can assume that perhaps maternal Wnt/STOP is also crucial for genome integrity maintenance in mammals by controlling mitotic microtubule assembly. 


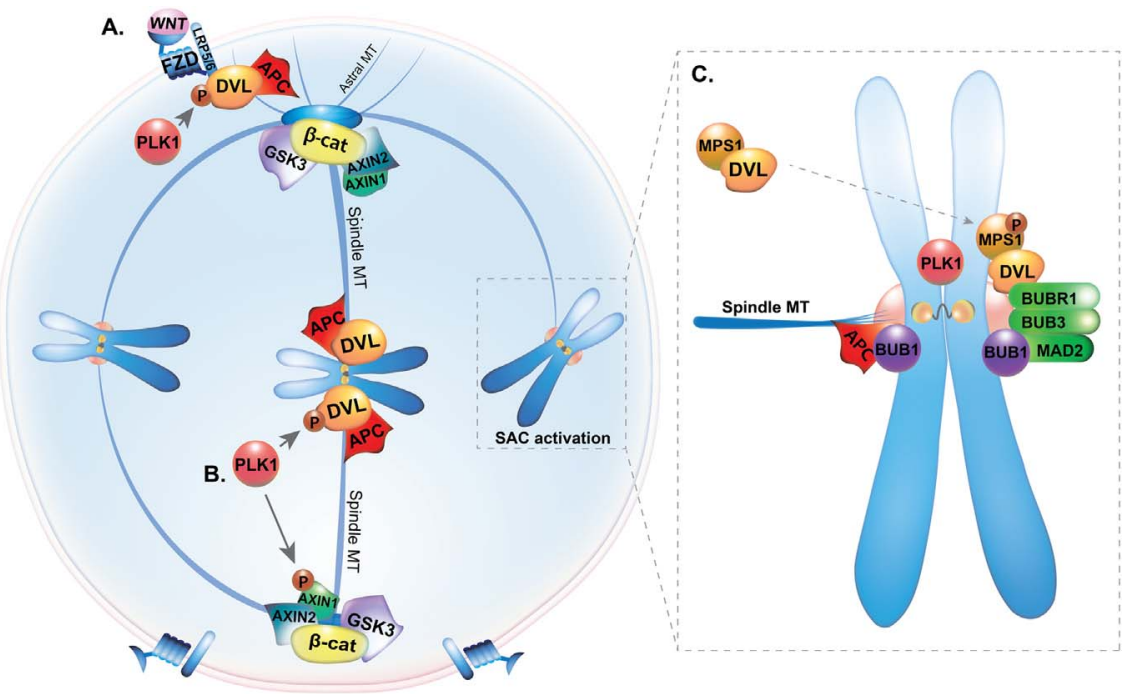

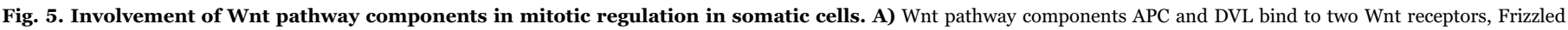

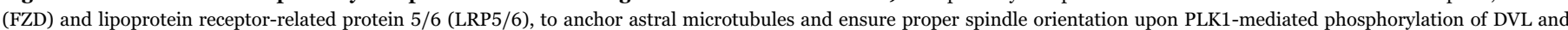

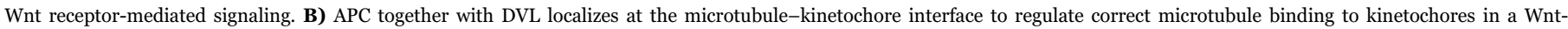
independent manner. C) DVL can also activate the spindle assembly checkpoint (SAC) by phosphorylating MPS1 and recruiting SAC components.

Although this assumption is still premature, it opens up new horizons to investigate novel potential roles of this pathway in early post-zygotic divisions in embryos.

\subsection{The MAPK pathway}

The mammalian MAP kinase signaling pathway is a highly conserved and complex signal transduction network that involves a cascade of protein phosphorylations, which culminate in activation of mitogen-activated protein kinases (MAPKs). Activated MAPK enzymes tightly coordinate cell proliferation, differentiation and survival, and include conventional MAPKs, such as extracellular signal-regulated kinases 1 and 2 (ERK1/2), c-Jun amino-terminal kinases 1-3 (JNK13), p38, and ERK5, as well as atypical MAPK enzymes with distinct regulation and function (reviewed in (Cargnello and Roux, 2011)). In somatic cells, MAPK/ERK signaling, also known as RAS-RAF-MEKERK pathway, contributes to proper spindle assembly, chromosome alignment and segregation, while p38-MAPK regulates cytoskeleton organization and participates in mitotic checkpoint regulation (Zhang and Liu, 2002). Because of important roles in different mitotic processes, it comes as no surprise that deregulation of the MAPK signaling has been implicated in neurodegenerative disorders and various types of cancer (Kim and Choi, 2010).

In oocyte biology, the MAPK pathway plays a crucial role in oocyte nuclear maturation by regulating meiotic processes. In mammals, the MOS kinase-mediated activation of MOS/MEK1/MAPK signaling cascade is necessary for maintaining meiotic metaphase II arrest. Maturing mouse Mos knockout oocytes fail to activate MAPK, which leads to distorted spindle and microtubule organization and spontaneous exit from meiosis. As a result, mice lacking MOS function often presented with ovarian teratomas due to parthenogenetic activation of the oocyte (Choi et al., 1996; Hashimoto et al., 1994; Verlhac et al., 1996). The importance of MAPK/ERK in meiotic arrest has also been corroborated by genome-wide analysis of gene expression and functional profiling in mice, demonstrating the lack of MAPK signaling in parthenote blastocysts (Liu et al., 2010). In addition, p38-MAPK is an important component of microtubule organizing centre (MTOC) in mouse oocytes that recruits $\gamma$-tubulin for spindle assembly, spindle pole formation and chromosome alignment (Ou et al., 2010). p38MAPK also phosphorylates MAPK-activated protein kinase 2 (MK2) that participates in meiotic cell cycle progression by regulating bipolar spindle stability, kMTs and chromosome segregation, while depletion of MK2 compromises both microtubule tension and SAC function, consequently leading to disorganized meiotic spindle and aneuploidy in mouse oocytes (Yuan et al., 2010).

In contrast to oocyte, the role of MAPK signaling in mammalian early preimplantation development is contradictory and remains elusive. Inhibition of MAPK pathway in sea urchin embryos leads to mitotic defects, such as aberrant spindle formation, chromosome misalignment and poor segregation, resulting in abnormal number of chromosomes in daughter cells (Zhang et al., 2005). Previous studies on mouse embryos also demonstrated a distinct mitotic M-phasespecific activation of MAP kinase signaling via RAS/RAF/MEK/ERK cascade (Haraguchi et al., 1998) that seems to be essential for cell cycle progression up to 8-cell stage (Maekawa et al., 2007). Intriguingly, the MAPK/ERK pathway is involved in protecting the the genomic integrity of mouse ESCs, as Erk depletion results in elevated rate of chromosome breakage and fusion, as well as telomere shortening possibly due to DNA damage (Chen et al., 2015). This suggests that MAPK signaling may have an important role in mitotic cell cycle progression, but whether the inhibition of MAPK/ERK signaling in mammals can lead to similar mitotic defects that were observed in sea urchin embryos remains to be tested.

It is worth noting that caution should be applied, as some differences in MAPK regulation and function can be observed between mammalian species. For instance, JNK- and p38-MAPK pathways play an important role in regulation of compaction and blastocyst formation in mouse preimplantation embryos (Bell and Watson, 2013; Maekawa et al., 2005). In the presence of p38-MAPK inhibitors, mouse embryos have delayed development, abnormal embryo morphology at the 8-16 cell stage and an increased rate of apoptosis in ICM (Sozen et al., 2015). In addition, p38-MAPK-deficient embryos also showed complete loss of filamentous actin (F-actin), an essential component of mitotic spindle (Natale et al., 2004; Paliga et al., 2005; Woolner et al., 2008). F-actin is involved in the cleavage furrow formation and its decreased function due long-term deficiency of p38-MAPK in mouse hepatocyte cells was shown to result in cytokinetic failure (Tormos et al., 2017). In turn, cytokinetic failure is associated with impaired human embryo compaction at 8-16 cell stage and the formation of multinucleated (polyploid) blastomeres (Iwata et al., 2014). As such, it is plausible that the embryonic phenotype observed in p38-MAPKdeficient mice may be a direct result of F-actin deprivation. In contrast, the development of bovine embryos was not significantly impaired upon inhibition of p38-MAPK, and only simultaneous absence of both 
p38-MAPK and MAPK/ERK signaling leads to similar developmental block as was observed in mouse embryos (Madan et al., 2005). Therefore, understanding the biological significance and regulatory mechanisms of MAPK pathway in mammals, including human, might provide novel insight into mitotic regulation of preimplantation development.

\subsection{The Hippo signaling pathway}

The evolutionally conserved Hippo pathway, crucial for maintaining organ growth through regulation of cell proliferation and apoptosis, was first discovered as a tumor suppressor pathway in Drosophila (Justice et al., 1995). The core kinase cascade components of the Hippo signaling pathway are MST1/MST2 and LATS1/LATS2 (Meng et al., 2015; Oh et al., 2009), while the transcriptional co-activators YAP and TAZ act as main downstream effectors (Piccolo et al., 2014). In mammals, the Hippo pathway is initially activated by TAO kinases that phosphorylate MST1/2, which subsequently recruit and phosphorylate LATS1/2 with the help of SAV1 and MOB1A/B (reviewed in (Meng et al., 2016)). In recent years the Hippo pathway has been mostly studied in mouse embryogenesis, where it modulates cellular differentiation and lineage segregation into TE and ICM through nuclear localization of YAP/TAZ in the mouse blastocyst (Fig. 6) (Anani et al., 2014; Leung and Zernicka-Goetz, 2013; Nishioka et al., 2009; Posfai et al., 2017). The Hippo MST/LATS/YAP signaling cascade might be conserved between species, but detailed information about this pathway in human is scarce.

Key Hippo signaling genes, such as YAP, TAZ, MST1/2, SAV1 and LATS1/2, are expressed in mouse and human ovarian follicles (Kawamura et al., 2013). Dynamic Hippo signaling has been implicated in mouse oocyte maturation, proliferation of granulosa cells and follicular atresia with main Hippo components showing differential expression at different time points of folliculogenesis (Xiang et al., 2015). Interestingly, in Drosophila oogenesis, Hippo pathway is required for oocyte polarization via microtubule-network orientation and the establishment of anterior-posterior axis. The disruption of
Hippo in fruit fly leads to aberrant follicle cell differentiation and loss of oocyte polarity due to disorganized microtubule formation, which may predispose the oocyte to chromosome missegregation (Polesello and Tapon, 2007; Yu et al., 2008). In contrast to Drosophila, oocytespecific deletion of YAP does not seem to affect oogenesis in mice, as mouse MII oocytes have well organized spindles (Yu et al., 2016). However, if lack of maternal YAP had no effect on mouse oocyte development, then mouse embryos already exhibited prolonged two-tofour cell stage progression compared to wild type, while doubleknockout embryos had severe morphokinetic defects that led to embryonic arrest before compaction. As such, it seems plausible to investigate the precise role of YAP in mammalian oocyte and early embryos.

In somatic cells, the activity of MST1 and MST2 was shown to increase during mitotic divisions, indicating their potential involvement in mitotic progression (Ling et al., 2008; Matallanas et al., 2008). Notably, MST1/MST2 deficiency results in severe developmental defects in early mouse embryos (Oh et al., 2009). Because downregulation of both MST1 and MST2 in human HeLa cells often causes chromosome misalignment and anaphase delay (Oh et al., 2010), the question arises whether early development of MST1/MST2-deficient embryos can also be compromised due to consequent accumulation of mitotic errors.

Similarly to MST1/MST2, LATS1 and LATS2 kinases also interact with various cell-cycle regulators and mitotic checkpoints (Tao et al., 1999; Yabuta et al., 2011; Yang et al., 2001), although the mechanisms of LATS1/2 activation are largely unclear. Nevertheless, the depletion of LATS2 in mouse embryonic fibroblasts leads to genomic instability, micronuclei formation, centrosome amplification and cytokinetic failure (McPherson et al., 2004). In addition, loss of LATS2 leads to downregulation of critical cell-cycle regulators, resulting in mitotic defects in somatic cells (Yabuta et al., 2007). Importantly, the depletion of key mitotic regulators in mouse and porcine embryos leads to developmental arrest and embryonic lethality by the blastocyst stage, possibly due to the accumulation of chromosomal defects (Diril et al., 2012; Wang and Kim, 2016). Collectively these data demonstrate that Hippo components may participate in mitotic processes and their abnormal

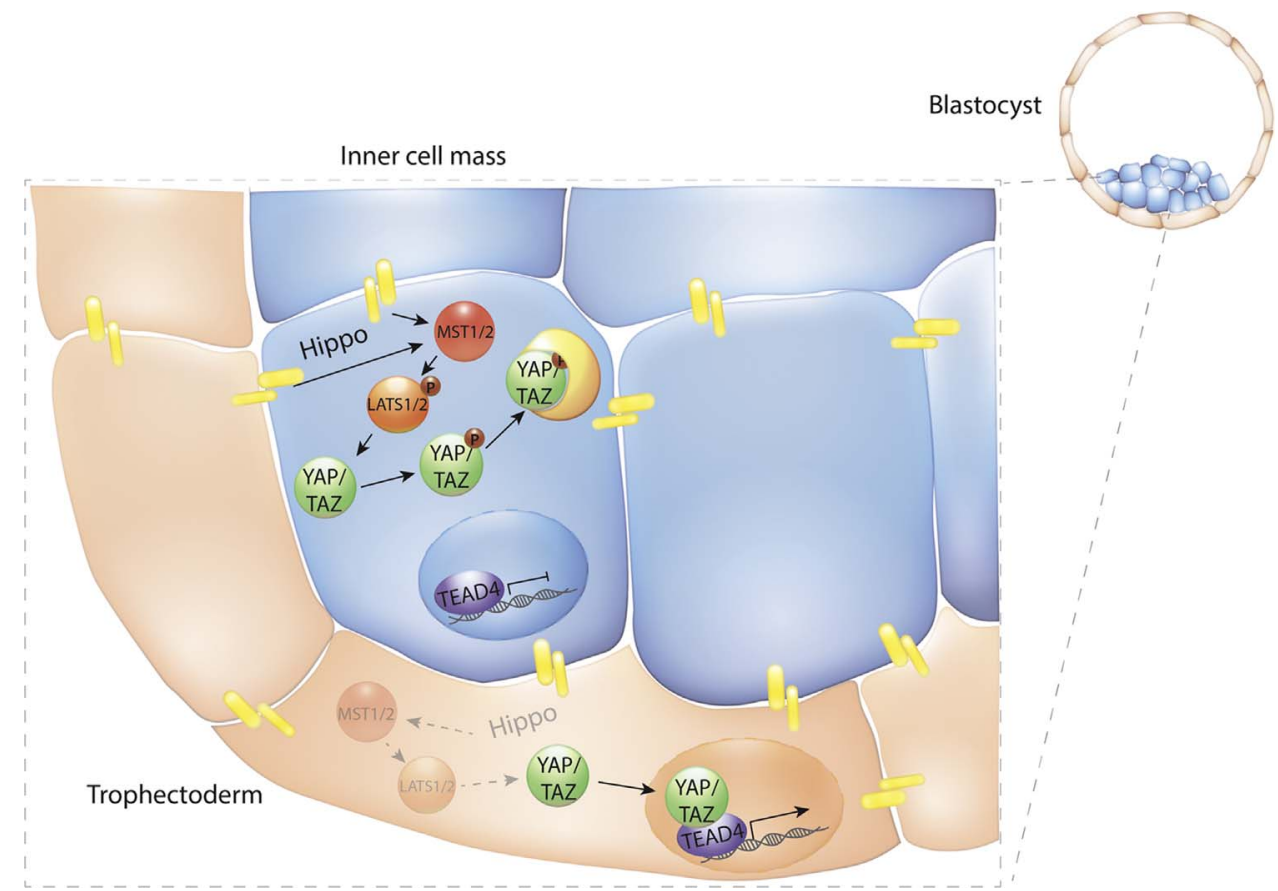

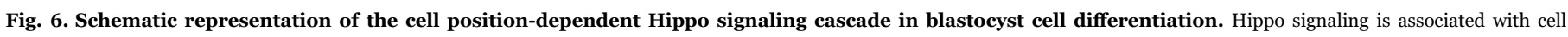

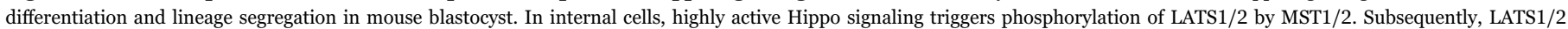

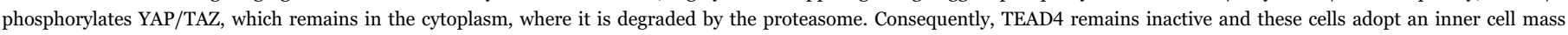

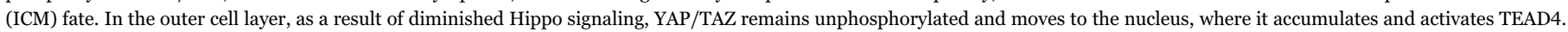
Consequently, active TEAD4 induces the activation of trophectoderm (TE)-specific factors and cells adopt TE fate. 
function may contribute to destabilizing genomic integrity. Further studies are required to enhance our understanding of the regulation of Hippo cascade components and to elucidate their potential role in genome stability in preimplantation embryos.

\section{Conclusion}

Research in the cancer field has highlighted some of the signaling pathways that may contribute to accurate mitotic progression by ensuring proper spindle formation and chromosome segregation during cell division. If deregulated, the same pathways can lead to genomic instability in somatic cancer cells. Moreover, embryonic stem cells with aberrant molecular signaling cannot be maintained due to self-renewal and chromosomal defects. However, currently it is not entirely clear whether or not altered signaling can lead to embryonic aneuploidy during preimplantation development. As such, this review had two main purposes: first, to give an overview on the origins of aneuploidy in preimplantation embryos; and second, to review the available human and animal data on the involvement of signaling pathways in promoting genomic stability; and to put this knowledge together into a speculative perspective on how alteration of molecular signaling may be responsible for aneuploidy in oocytes and early embryos. Although the robust links between molecular pathways and CIN in embryos are yet to be determined, different molecular signaling components have already been associated with genome integrity in both oocytes and embryos. Despite the fact that the chromosome segregation errors are unlikely to be linked to only one particular pathway, our understanding on the regulation of intracellular signaling within the mammalian embryo may likely lead to a better understanding of aetiology of embryonic aneuploidy, a question that has a direct clinical implication. However, caution is warranted, when extrapolating the data obtained from different animal models, as they may not always reflect the nature of human embryogenesis. We anticipate that future research will pursuit important fundamental questions that will unravel in greater detail (i) the role of signaling pathways, if any, in ensuring genomic stability of early embryos, (ii) the role of maternal factors in first postzygotic mitotic processes and (iii) how maternal age and in vitro conditions may lead to altered expression of genes involved in oocyte and early embryo signaling.

\section{Acknowledgements}

We are grateful to Dr. Aspasia Destouni for her critical revision and valuable feedback on the manuscript. This study was funded by Estonian Ministry of Education and Research (Grant IUT34-16); Enterprise Estonia (Grant EU48695); the EU-FP7 Eurostars Program (Grant NOTED, EU41564); the EU-FP7 Marie Curie IndustryAcademia Partnerships and Pathways (IAPP, Grant SARM, EU324509); Horizon 2020 Innovation Programme (WIDENLIFE, 692065); and grant from the University of Granada (UGR-INC16-48) (Incorporación de jóvenes doctores) and grant from the Spanish Ministry of Science and Innovation - MINECO - (RYC-2016-21199 and Grant ENDORE, SAF2017-87526-R).

\section{References}

Acebron, S.P., et al., 2014. Mitotic wnt signaling promotes protein stabilization and regulates cell size. Mol. Cell 54, 663-674.

Acevedo, N., et al., 2007. Glycogen synthase kinase-3 regulation of chromatin segregation and cytokinesis in mouse preimplantation embryos. Mol. Reprod. Dev. 74, 178-188.

Adiga, S.K., et al., 2007. Delayed and stage specific phosphorylation of H2AX during preimplantation development of gamma-irradiated mouse embryos. Reproduction $133,415-422$.

Altmäe, S., et al., 2010. Endometrial gene expression analysis at the time of embryo implantation in women with unexplained infertility. Mol. Hum. Reprod. 16, $178-187$.

Anani, S., et al., 2014. Initiation of Hippo signaling is linked to polarity rather than to cell position in the pre-implantation mouse embryo. Development 141, 2813-2824.
Artus, J., et al., 2006. The cell cycle of early mammalian embryos: lessons from genetic mouse models. Cell Cycle 5, 499-502.

Assou, S., et al., 2010. Dynamic changes in gene expression during human early embryo development: from fundamental aspects to clinical applications. Hum. Reprod. Update 17, 272-290.

Augustin, I., et al., 2017. Autocrine Wnt regulates the survival and genomic stability of embryonic stem cells. Sci. Signal., 10.

Bakhoum, S.F., et al., 2014. DNA-damage response during mitosis induces wholechromosome missegregation. Cancer Discov. 4, 1281-1289.

Bazrgar, M., et al., 2014. DNA repair signalling pathway genes are overexpressed in poorquality pre-implantation human embryos with complex aneuploidy. Eur. J. Obstet. Gynecol. Reprod. Biol. 175, 152-156.

Bell, C.E., Watson, A.J., 2013. p38 MAPK regulates cavitation and tight junction function in the mouse blastocyst. PLoS One 8, e59528.

Bolton, H., et al., 2016. Mouse model of chromosome mosaicism reveals lineage-specific depletion of aneuploid cells and normal developmental potential. Nat. Commun. 7, 11165.

Bourke, E., et al., 2007. DNA damage induces Chk1-dependent centrosome amplification. EMBO Rep. 8, 603-609.

Brunet, S., Verlhac, M.H., 2011. Positioning to get out of meiosis: the asymmetry of division. Hum. Reprod. Update 17, 68-75.

Burton, A., Torres-Padilla, M.E., 2014. Chromatin dynamics in the regulation of cell fate allocation during early embryogenesis. Nat. Rev. Mol. Cell Biol. 15, 723-734.

Cargnello, M., Roux, P.P., 2011. Activation and function of the MAPKs and their substrates, the MAPK-activated protein kinases. Microbiol. Mol. Biol. Rev. 75 , $50-83$.

Carroll, J., Marangos, P., 2013. The DNA damage response in mammalian oocytes. Front. Genet. 4, 117.

Celeste, A., et al., 2002. Genomic instability in mice lacking histone H2AX. Science 296, 922-927.

Chamayou, S., et al., 2013. The use of morphokinetic parameters to select all embryos with full capacity to implant. J. Assist. Reprod. Genet. 30, 703-710.

Chavez, S.L., et al., 2012. Dynamic blastomere behaviour reflects human embryo ploidy by the four-cell stage. Nat. Commun. 3, 1251.

Chen, H., et al., 2015. Erk signaling is indispensable for genomic stability and selfrenewal of mouse embryonic stem cells. Proc. Natl. Acad. Sci. USA 112, E5936-E5943.

Chiang, T., et al., 2011. Age-dependent susceptibility of chromosome cohesion to premature separase activation in mouse oocytes. Biol. Reprod. 85, 1279-1283.

Chila, R., et al., 2013. Chk1-Mad2 interaction: a crosslink between the DNA damage checkpoint and the mitotic spindle checkpoint. Cell Cycle 12, 1083-1090.

Choi, T., et al., 1996. The Mos/mitogen-activated protein kinase (MAPK) pathway regulates the size and degradation of the first polar body in maturing mouse oocytes. Proc. Natl. Acad. Sci. USA 93, 7032-7035.

Chow, J.F., et al., 2014. Array comparative genomic hybridization analyses of all blastomeres of a cohort of embryos from young IVF patients revealed significant contribution of mitotic errors to embryo mosaicism at the cleavage stage. Reprod. Biol. Endocrinol. 12, 105

Cimini, D., et al., 2001. Merotelic kinetochore orientation is a major mechanism of aneuploidy in mitotic mammalian tissue cells. J. Cell Biol. 153, 517-527.

Clift, D., Schuh, M., 2013. Restarting life: fertilization and the transition from meiosis to mitosis. Nat. Rev. Mol. Cell Biol. 14, 549-562.

Crasta, K., et al., 2012. DNA breaks and chromosome pulverization from errors in mitosis. Nature 482, 53-58.

Destouni, A., Vermeesch, J.R., 2017. How can zygotes segregate entire parental genomes into distinct blastomeres? The zygote metaphase revisited. Bioessays, 39 .

Destouni, A., et al., 2016. Zygotes segregate entire parental genomes in distinct blastomere lineages causing cleavage-stage chimerism and mixoploidy. Genome Res. $26,567-578$.

Diril, M.K., et al., 2012. Cyclin-dependent kinase 1 (Cdk1) is essential for cell division and suppression of DNA re-replication but not for liver regeneration. Proc. Natl. Acad. Sci. USA 109, 3826-3831.

Dupont, C., et al., 2009. Chromosomal instability in rhesus macaque preimplantation embryos. Fertil. Steril. 91, 1230-1237.

Dupont, C., et al., 2010. Incidence of chromosomal mosaicism in morphologically normal nonhuman primate preimplantation embryos. Fertil. Steril. 93, 2545-2550.

Edwards, M.J., et al., 1994. Mixoploidy in humans: two surviving cases of diploidtetraploid mixoploidy and comparison with diploid-triploid mixoploidy. Am. J. Med. Genet. 52, 324-330.

Fear, J.M., Hansen, P.J., 2011. Developmental changes in expression of genes involved in regulation of apoptosis in the bovine preimplantation embryo. Biol. Reprod. 84, 43-51.

Fledel-Alon, A., et al., 2009. Broad-scale recombination patterns underlying proper disjunction in humans. PLoS Genet. 5, e1000658.

Fodde, R., et al., 2001. Mutations in the APC tumour suppressor gene cause chromosomal instability. Nat. Cell Biol. 3, 433-438.

Franasiak, J.M., et al., 2014. The nature of aneuploidy with increasing age of the female partner: a review of 15,169 consecutive trophectoderm biopsies evaluated with comprehensive chromosomal screening. Fertil. Steril. 101, 656-663.e1.

Gawecka, J.E., et al., 2013. Mouse zygotes respond to severe sperm DNA damage by delaying paternal DNA replication and embryonic development. PLoS One 8, e56385

Greco, E., et al., 2015. Healthy babies after intrauterine transfer of mosaic aneuploid blastocysts. N. Engl. J. Med. 373, 2089-2090.

Hadjihannas, M.V., et al., 2010. Conductin/axin2 and Wnt signalling regulates centrosome cohesion. EMBO Rep. 11, 317-324. 
Hadjihannas, M.V., et al., 2006. Aberrant Wnt/beta-catenin signaling can induce chromosomal instability in colon cancer. Proc. Natl. Acad. Sci. USA 103, 10747-10752.

Han, Z., et al., 2010. Early transcription from the maternal genome controlling blastomere integrity in mouse two-cell-stage embryos. Am. J. Physiol. Cell Physiol. 298, C1235-C1244.

Haraguchi, S., et al., 1998. MAP kinase cascade, but not ERKs, activated during early cleavage of mouse embryos. Mol. Reprod. Dev. 51, 148-155.

Hardy, K., et al., 1993. Binucleate blastomeres in preimplantation human embryos in vitro: failure of cytokinesis during early cleavage. J. Reprod. Fertil. 98, $549-558$.

Harwood, B.N., et al., 2008. Members of the WNT signaling pathways are widely expressed in mouse ovaries, oocytes, and cleavage stage embryos. Dev. Dyn. 237, $1099-1111$.

Hashimoto, N., et al., 1994. Parthenogenetic activation of oocytes in c-mos-deficient mice. Nature 370, 68-71.

Hassold, T., Hunt, P., 2001. To err (meiotically) is human: the genesis of human aneuploidy. Nat. Rev. Genet. 2, 280-291.

Hayashi, M.T., Karlseder, J., 2013. DNA damage associated with mitosis and cytokinesis failure. Oncogene 32, 4593-4601.

He, X.Q., et al., 2016. Axin-1 regulates meiotic spindle organization in mouse oocytes. PLoS One 11, e0157197.

Hernandez Gifford, J.A., 2015. The role of WNT signaling in adult ovarian folliculogenesis. Reproduction 150, R137-R148.

Hirose, Y., et al., 2011. Chiasmata promote monopolar attachment of sister chromatids and their co-segregation toward the proper pole during meiosis I. PLoS Genet. 7, e1001329.

Hlinka, D., et al., 2012. Time-lapse cleavage rating predicts human embryo viability. Physiol. Res. 61, 513-525.

Horcajadas, J.A., et al., 2007. Wide genomic analysis of human endometrial receptivity: new times, new opportunities. Hum. Reprod. Update 13, 77-86.

Huang, Y.L., et al., 2015. Maternal Wnt/STOP signaling promotes cell division during early Xenopus embryogenesis. Proc. Natl. Acad. Sci. USA 112, 5732-5737.

Hut, H.M., et al., 2003. Centrosomes split in the presence of impaired DNA integrity during mitosis. Mol. Biol. Cell 14, 1993-2004.

Iwata, K., et al., 2014. Analysis of compaction initiation in human embryos by using time-lapse cinematography. J. Assist. Reprod. Genet. 31, 421-426.

Jacobs, K., et al., 2017. Mitotic spindle disruption in human preimplantation embryos activates the spindle assembly checkpoint but not apoptosis until day 5 of development. Mol. Hum. Reprod..

Jaroudi, S., SenGupta, S., 2007. DNA repair in mammalian embryos. Mutat. Res. 635 $53-77$.

Jarvela, I.E., et al., 1993. 46,XX/69,XXX diploid-triploid mixoploidy with hypothyroidism and precocious puberty. J. Med. Genet. 30, 966-967.

Justice, R.W., et al., 1995. The Drosophila tumor suppressor gene warts encodes a homolog of human myotonic dystrophy kinase and is required for the control of cell shape and proliferation. Genes Dev. 9, 534-546.

Kaplan, K.B., et al., 2001. A role for the Adenomatous Polyposis Coli protein in chromosome segregation. Nat. Cell Biol. 3, 429-432.

Kawamura, K., et al., 2013. Hippo signaling disruption and Akt stimulation of ovarian follicles for infertility treatment. Proc. Natl. Acad. Sci. USA 110, 17474-17479.

Kiessling, A.A., 2010. Timing is everything in the human embryo. Nat. Biotechnol. 28, $1025-1026$.

Kiessling, A.A., et al., 2009. Evidence that human blastomere cleavage is under unique cell cycle control. J. Assist. Reprod. Genet. 26, 187-195.

Kiessling, A.A., et al., 2010. Genome-wide microarray evidence that 8-cell human blastomeres over-express cell cycle drivers and under-express checkpoints. J. Assist. Reprod. Genet. 27, 265-276.

Kikuchi, K., et al., 2010. Dishevelled, a Wnt signalling component, is involved in mitotic progression in cooperation with Plk1. Embo J. 29, 3470-3483.

Kim, E.K., Choi, E.J., 2010. Pathological roles of MAPK signaling pathways in human diseases. Biochim. Biophys. Acta 1802, 396-405.

Knofler, M., Pollheimer, J., 2013. Human placental trophoblast invasion and differentiation: a particular focus on Wnt signaling. Front. Genet. 4, 190.

Komiya, Y., Habas, R., 2008. Wnt signal transduction pathways. Organogenesis 4, 68-75.

Ladstatter, S., Tachibana-Konwalski, K., 2016. A surveillance mechanism ensures repair of DNA lesions during zygotic reprogramming. Cell 167 (1774-1787), e13.

Leung, C.Y., Zernicka-Goetz, M., 2013. Angiomotin prevents pluripotent lineage differentiation in mouse embryos via Hippo pathway-dependent and -independent mechanisms. Nat. Commun. 4, 2251.

Lightfoot, D.A., et al., 2006. The fate of mosaic aneuploid embryos during mouse development. Dev. Biol. 289, 384-394.

Ling, P., et al., 2008. Biosignaling of mammalian Ste20-related kinases. Cell Signal. 20, $1237-1247$.

Lister, L.M., et al., 2010. Age-related meiotic segregation errors in mammalian oocytes are preceded by depletion of cohesin and Sgo2. Curr. Biol. 20, 1511-1521.

Liu, N., et al., 2010. Genome-wide gene expression profiling reveals aberrant MAPK and Wnt signaling pathways associated with early parthenogenesis. J. Mol. Cell Biol. 2, 333-344.

Liu, Q., et al., 2000. Chk1 is an essential kinase that is regulated by Atr and required for the G(2)/M DNA damage checkpoint. Genes Dev. 14, 1448-1459.

Ly, P., et al., 2017. Selective Y centromere inactivation triggers chromosome shattering in micronuclei and repair by non-homologous end joining. Nat. Cell Biol. 19, 68-75.

Ma, Y., et al., 2010. The relationship between early embryo development and tumourigenesis. J. Cell Mol. Med. 14, 2697-2701.
MacDonald, B.T., et al., 2009. Wnt/beta-catenin signaling: components, mechanisms, and diseases. Dev. Cell 17, 9-26.

Madan, P., et al., 2005. Mitogen-activated protein kinase (MAPK) blockade of bovine preimplantation embryogenesis requires inhibition of both p38 and extracellular signal-regulated kinase (ERK) pathways. Reproduction 130, 41-51.

Maekawa, M., et al., 2007. Requirement for ERK MAP kinase in mouse preimplantation development. Development 134, 2751-2759.

Maekawa, M., et al., 2005. Requirement of the MAP kinase signaling pathways for mouse preimplantation development. Development 132, 1773-1783.

Mantikou, E., et al., 2012. Molecular origin of mitotic aneuploidies in preimplantation embryos. Biochim. Biophys. Acta 1822, 1921-1930.

Marechal, A., Zou, L., 2013. DNA damage sensing by the ATM and ATR kinases. Cold Spring Harb. Perspect. Biol., 5.

Matallanas, D., et al., 2008. A Hippo in the ointment: MST signalling beyond the fly. Cell Cycle 7, 879-884.

Maurer, M., et al., 2015. Chromosomal Aneuploidies and Early Embryonic Developmental Arrest. Int. J. Fertil Steril. 9, 346-353.

McPherson, J.P., et al., 2004. Lats2/Kpm is required for embryonic development, proliferation control and genomic integrity. Embo J. 23, 3677-3688.

Meng, Z., et al., 2016. Mechanisms of Hippo pathway regulation. Genes Dev. 30, 1-17.

Meng, Z., et al., 2015. MAP4K family kinases act in parallel to MST1/2 to activate LATS1/2 in the Hippo pathway. Nat. Commun. 6, 8357.

Mertzanidou, A., et al., 2012. Microarray analysis reveals abnormal chromosomal complements in over $70 \%$ of 14 normally developing human embryos. Hum. Reprod. $28,256-264$.

Miller, J.R., 2002. The Wnts. Genome Biol., 3, (REVIEWS3001).

Munne, S., et al., 1995. Embryo morphology, developmental rates, and maternal age are correlated with chromosome abnormalities. Fertil. Steril. 64, 382-391.

Nagaoka, S.I., et al., 2011. Oocyte-specific differences in cell-cycle control create an innate susceptibility to meiotic errors. Curr. Biol. 21, 651-657.

Naillat, F., et al., 2010. Wnt4/5a signalling coordinates cell adhesion and entry into meiosis during presumptive ovarian follicle development. Hum. Mol. Genet. 19, 1539-1550.

Nakagawa, S., FitzHarris, G., 2017. Intrinsically defective microtubule dynamics contribute to age-related chromosome segregation errors in mouse oocyte meiosis-I. Curr. Biol. 27, 1040-1047.

Natale, D.R., et al., 2004. p38 MAPK signaling during murine preimplantation development. Dev. Biol. 268, 76-88.

Niehrs, C., Acebron, S.P., 2012. Mitotic and mitogenic Wnt signalling. Embo J. 31, 2705-2713.

Nishioka, N., et al., 2009. The Hippo signaling pathway components Lats and Yap pattern Tead4 activity to distinguish mouse trophectoderm from inner cell mass. Dev. Cell $16,398-410$.

O'Shea, L.C., et al., 2012. Developmental competence in oocytes and cumulus cells: candidate genes and networks. Syst. Biol. Reprod. Med. 58, 88-101.

Oh, H.J., et al., 2010. MST1 limits the kinase activity of aurora B to promote stable kinetochore-microtubule attachment. Curr. Biol. 20, 416-422.

Oh, S., et al., 2009. Crucial role for Mst1 and Mst2 kinases in early embryonic development of the mouse. Mol. Cell Biol. 29, 6309-6320.

Ohkura, H., 2015. Meiosis: an overview of key differences from mitosis. Cold Spring Harb. Perspect. Biol., 7.

Orr, B., Compton, D.A., 2013. A double-edged sword: how oncogenes and tumor suppressor genes can contribute to chromosomal instability. Front. Oncol. 3, 164.

Ottolini, C.S., et al., 2015. Karyomapping identifies second polar body DNA persisting to the blastocyst stage: implications for embryo biopsy. Reprod. Biomed. Online 31, $776-782$.

Ou, X.H., et al., 2010. p38alpha MAPK is a MTOC-associated protein regulating spindle assembly, spindle length and accurate chromosome segregation during mouse oocyte meiotic maturation. Cell Cycle 9, 4130-4143.

Paliga, A.J., et al., 2005. p38 mitogen-activated protein kinase (MAPK) first regulates filamentous actin at the 8-16-cell stage during preimplantation development. Biol. Cell 97, 629-640.

Paull, T.T., et al., 2000. A critical role for histone H2AX in recruitment of repair factors to nuclear foci after DNA damage. Curr. Biol. 10, 886-895.

Peddibhotla, S., et al., 2009. The DNA-damage effector checkpoint kinase 1 is essential for chromosome segregation and cytokinesis. Proc. Natl. Acad. Sci. USA 106, 5159-5164.

Piccolo, S., et al., 2014. The biology of YAP/TAZ: hippo signaling and beyond. Physiol. Rev. 94, 1287-1312.

Polesello, C., Tapon, N., 2007. Salvador-warts-hippo signaling promotes Drosophila posterior follicle cell maturation downstream of notch. Curr. Biol. 17, 1864-1870.

Posfai, E., et al., 2017. Position- and Hippo signaling-dependent plasticity during lineage segregation in the early mouse embryo. Elife, 6 .

Rambags, B.P., et al., 2005. Numerical chromosomal abnormalities in equine embryos produced in vivo and in vitro. Mol. Reprod. Dev. 72, 77-87.

Ruan, K., et al., 2012. PLK1 interacts and phosphorylates Axin that is essential for proper centrosome formation. PLoS One 7, e49184.

Salumets, A., et al., 2002. Influence of oocytes and spermatozoa on early embryonic development. Fertil. Steril. 78, 1082-1087.

Schuh, M., Ellenberg, J., 2007. Self-organization of MTOCs replaces centrosome function during acentrosomal spindle assembly in live mouse oocytes. Cell 130, 484-498.

Silkworth, W.T., Cimini, D., 2012. Transient defects of mitotic spindle geometry and chromosome segregation errors. Cell Div. 7, 19.

Smith, J., et al., 2010. The ATM-Chk2 and ATR-Chk1 pathways in DNA damage signaling and cancer. Adv. Cancer Res. 108, 73-112. 
Sozen, B., et al., 2015. The p38 MAPK signalling pathway is required for glucose metabolism, lineage specification and embryo survival during mouse preimplantation development. Mech. Dev. 138 (Pt 3), 375-398.

Staessen, C., Van Steirteghem, A.C., 1997. The chromosomal constitution of embryos developing from abnormally fertilized oocytes after intracytoplasmic sperm injection and conventional in-vitro fertilization. Hum. Reprod. 12, 321-327.

Stensen, M.H., et al., 2014. Fragmentation of human cleavage-stage embryos is related to the progression through meiotic and mitotic cell cycles. Fertil. Steril. 103, 374-381.e4.

Stolz, A., et al., 2015. Wnt-mediated protein stabilization ensures proper mitotic microtubule assembly and chromosome segregation. EMBO Rep. 16, 490-499.

Sun, S.C., Kim, N.H., 2012. Spindle assembly checkpoint and its regulators in meiosis. Hum. Reprod. Update 18, 60-72.

Takai, H., et al., 2000. Aberrant cell cycle checkpoint function and early embryonic death in Chk1(-/-) mice. Genes Dev. 14, 1439-1447.

Tao, W., et al., 1999. Human homologue of the Drosophila melanogaster lats tumour suppressor modulates CDC2 activity. Nat. Genet. 21, 177-181.

Tepekoy, F., et al., 2015. The role of Wnt signaling members in the uterus and embryo during pre-implantation and implantation. J. Assist. Reprod. Genet. 32, $337-346$.

Thompson, S.L., Compton, D.A., 2011. Chromosome missegregation in human cells arises through specific types of kinetochore-microtubule attachment errors. Proc. Natl. Acad. Sci. USA 108, 17974-17978.

Titus, S., et al., 2013. Impairment of BRCA1-related DNA double-strand break repair leads to ovarian aging in mice and humans. Sci. Transl. Med. 5, 172ra21.

Tormos, A.M., et al., 2017. p38alpha Regulates actin cytoskeleton and cytokinesis in hepatocytes during development and aging. PLoS One 12, e0171738.

Tsuiko, O., et al., 2017. Genome stability of bovine in vivo-conceived cleavage-stage embryos is higher compared to in vitro-produced embryos. Hum. Reprod. 32, 2348-2357.

Tsutsumi, M., et al., 2014. Age-related decrease of meiotic cohesins in human oocytes. PLoS One 9, e96710.

Usongo, M., et al., 2012. beta-Catenin/Tcf signaling in murine oocytes identifies nonovulatory follicles. Reproduction 144, 669-676.

Valenta, T., et al., 2012. The many faces and functions of beta-catenin. Embo J. 31, 2714-2736.

van de Werken, C., et al., 2015. Chromosome segregation regulation in human zygotes: altered mitotic histone phosphorylation dynamics underlying centromeric targeting of the chromosomal passenger complex. Hum. Reprod. 30, 2275-2291.

Vanneste, E., et al., 2009. Chromosome instability is common in human cleavage-stage embryos. Nat. Med. 15, 577-583.

Vera-Rodriguez, M., et al., 2015. Prediction model for aneuploidy in early human embryo development revealed by single-cell analysis. Nat. Commun. 6, 7601.

Verlhac, M.H., et al., 1996. Mos is required for MAP kinase activation and is involved in microtubule organization during meiotic maturation in the mouse. Development $122,815-822$.

Viuff, D., et al., 2000. Chromosome aberrations in in vitro-produced bovine embryos at days 2-5 post-insemination. Biol. Reprod. 63, 1143-1148.

Viuff, D., et al., 2001. Chromosomal abnormalities and developmental kinetics in in vivodeveloped cattle embryos at days 2-5 after ovulation. Biol. Reprod. 65, 204-208.

Voet, T., et al., 2011a. Breakage-fusion-bridge cycles leading to inv dup del occur in human cleavage stage embryos. Hum. Mutat. 32, 783-793.

Voet, T., et al., 2011b. The human cleavage stage embryo is a cradle of chromosomal rearrangements. Cytogenet. Genome Res. 133, 160-168.

Vrooman, L.A., et al., 2014. Evidence for paternal age-related alterations in meiotic chromosome dynamics in the mouse. Genetics 196, 385-396.
Wakefield, J.G., et al., 2003. A role for glycogen synthase kinase-3 in mitotic spindle dynamics and chromosome alignment. J. Cell Sci. 116, 637-646.

Wang, H., Kim, N.H., 2016. CDK2 is required for the DNA damage response during porcine early embryonic development. Biol. Reprod. 95, 31.

Wang, H., et al., 2015. Effect of ATM and HDAC inhibition on etoposide-induced DNA damage in porcine early preimplantation embryos. PLoS One 10, e0142561.

Wang, Q.T., et al., 2004. A genome-wide study of gene activity reveals developmental signaling pathways in the preimplantation mouse embryo. Dev. Cell 6, 133-144.

Wang, X., et al., 2017. Transcriptome analyses of rhesus monkey preimplantation embryos reveal a reduced capacity for DNA double-strand break repair in primate oocytes and early embryos. Genome Res. 27, 567-579.

Webster, A., Schuh, M., 2016. Mechanisms of aneuploidy in human eggs. Trends Cell Biol. 27, 55-68.

Wells, D., et al., 2005. Expression of genes regulating chromosome segregation, the cell cycle and apoptosis during human preimplantation development. Hum. Reprod. 20, 1339-1348.

Woolner, S., et al., 2008. Myosin-10 and actin filaments are essential for mitotic spindle function. J. Cell Biol. 182, 77-88.

Xiang, C., et al., 2015. Hippo signaling pathway reveals a spatio-temporal correlation with the size of primordial follicle pool in mice. Cell Physiol. Biochem. 35, 957-968.

Yabuta, N., et al., 2011. The tumor suppressor Lats2 is pivotal in Aurora A and Aurora B signaling during mitosis. Cell Cycle 10, 2724-2736.

Yabuta, N., et al., 2007. Lats2 is an essential mitotic regulator required for the coordination of cell division. J. Biol. Chem. 282, 19259-19271.

Yamamoto, K., et al., 2012. Kinase-dead ATM protein causes genomic instability and early embryonic lethality in mice. J. Cell Biol. 198, 305-313.

Yamazawa, K., et al., 2010. Parthenogenetic chimaerism/mosaicism with a Silver-Russell syndrome-like phenotype. J. Med. Genet. 47, 782-785.

Yang, X., et al., 2001. Human homologue of Drosophila lats, LATS1, negatively regulate growth by inducing $\mathrm{G}(2) / \mathrm{M}$ arrest or apoptosis. Oncogene 20, 6516-6523.

Yu, C., et al., 2016. Oocyte-expressed yes-associated protein is a key activator of the early zygotic genome in mouse. Cell Res. 26, 275-287.

Yu, J., et al., 2008. The hippo pathway promotes Notch signaling in regulation of cell differentiation, proliferation, and oocyte polarity. PLoS One 3, e1761.

Yuan, J., et al., 2010. MAPK-activated protein kinase 2 is required for mouse meiotic spindle assembly and kinetochore-microtubule attachment. PLoS One 5, e11247.

Yun, Y., et al., 2014. Reduced ability to recover from spindle disruption and loss of kinetochore spindle assembly checkpoint proteins in oocytes from aged mice. Cell Cycle 13, 1938-1947.

Zachos, G., et al., 2007. Chk1 is required for spindle checkpoint function. Dev. Cell 12, 247-260.

Zamani Esteki, M., et al., 2015. Concurrent whole-genome haplotyping and copy-number profiling of single cells. Am. J. Hum. Genet. 96, 894-912.

Zhang, D., et al., 2015. Increased DNA damage and repair deficiency in granulosa cells are associated with ovarian aging in rhesus monkey. J. Assist. Reprod. Genet. 32, 1069-1078.

Zhang, W., Liu, H.T., 2002. MAPK signal pathways in the regulation of cell proliferation in mammalian cells. Cell Res. 12, 9-18.

Zhang, W.L., et al., 2005. A MAPK pathway is involved in the control of mitosis after fertilization of the sea urchin egg. Dev. Biol. 282, 192-206.

Zhang, Y., et al., 2007. Signaling pathways and preimplantation development of mammalian embryos. Febs J. 274, 4349-4359.

Zhang, Z., et al., 2017. Plk1 inhibition leads to a failure of mitotic division during the first mitotic division in pig embryos. J. Assist. Reprod. Genet. 34, 399-407.

Zudova, D., et al., 2003. Aneuploidy detection in porcine embryos using fluorescence in situ hybridization. Cytogenet. Genome Res. 102, 179-183. 